Ю.Г. Выхованец, С.М. Тетюра, А.Н. Черняк, П.А. Алешечкин

ОЦЕНКА ОБЕЗБОЛИВАЮЩЕГО ЭФФЕКТА И ВЛИЯЮЩИХ НА НЕГО ФАКТОРОВ ПРИ ВОЗДЕЙСТВИИ ЭКСТРЕМАЛЬНО НИЗКОЙ ТЕМПЕРАТУРЫ У БОЛЬНЫХ С ДЕФОРМИРУЮЩИМИ ДОРСОПАТИЯМИ

\author{
Донецкий национальный медицинский университет имени М. Горького, \\ 83003, Украина, Донецк, пр. Ильича, 16. E-mail: tsergmich@mail.ru
}

Цель: оценить обезболивающий эффект и влияющие на него факторы при воздействии экстремально низкой температуры в условиях криокамеры для общей воздушной криотерапии. Материалы и методы: в исследованиях добровольно принимали участие 335 пациентов (144 мужчины и 191 женщина) в возрасте от 20 до 72 лет с патологией опорно-двигательного аппарата (деформирующие дорсопатии) с доминирующим болевым синдромом. Для создания экстремально низкой температуры в исследовании применялась воздушная криогенная камера. Результаты: анализ кривых вероятности отсутствия обезболивающего эффекта показал, что у 50,0\% исследуемых ориентировочно при ежедневных криотерапевтических процедурах выраженный обезболивающий эффект развивается после $20 \pm 1,5$ сеанса. Выводы: у 95\% больных с деформирующими дорсопатиями максимальный лечебный эффект развивается не ранее 30-го дня при ежедневном воздействии экстремально низкой температуры. Влияние возрастных, гендерных и сезонных факторов на эффективность лечения у данного контингента больных статистически невыявлено (p>0,05).

Ключевые слова: криотерапия, экстремально низкая температура, обезболивающий эффект, дорсопатии.

Y. G. Vyhovanets, S. M. Tetyura, A.N.Cherniak, P.A. Alyoshechkin

\title{
ESTIMATION OF ANALGETIC EFFECT AT EXTREMELY LOW TEMPERATURE EXPOSURE IN PATIENTS WITH DEFORMING DORSOPATHIES
}

\author{
M.Gorky Donetsk National Medical University, \\ 16 Illicha av., Donetsk, 83003, Ukraine.E-mail: tsergmich@mail.ru
}

\begin{abstract}
Objective: estimate of analgetic effect at extremely low temperature in the conditions of cryochamber for general air cryotherapy. Materials and methods: 335 patients (144 males and 191 females) in age from 20 to 72 years with pathology of locomotorium (deforming dorsopathies) took part in researches. Researches were conducted with the use of cryochamber for creation extremely low temperature. Results: the analysis of curves of probability of absence of analgetic effect showed that approximately $50,0 \%$ of patients had the lasting analgetic effect after $20 \pm 1,50$ session of treatment. Conclusions: for achievement of maximal analgetic effect at $95 \%$ patients approximately 30 valuable sessions of general air cryotherapy in the cryochambe required. Influences of age, gender and seasons on efficiency of antinociceptive action at this contingent of patients statistically was not detected $(\mathrm{p}>0,05)$.

Keywords: cryotherapy, extremely subzero temperature, analgetic effect, dorsopathy.
\end{abstract}




\section{Введение}

$\mathrm{O}$ громное количество проведенных в последние годы научных исследований доказало высокую клиническую эффективность кратковременного воздействия экстремально низкой температуры на организм человека $[1,2]$. Результаты таких исследований в настоящее время активно используются в центрах восстановительного лечения и реабилитации пациентов с разнообразной патологией опорно-двигательного аппарата (артриты, артрозы, полиартриты, остеохондроз и др.), в дерматологических клиниках для лечения резистентных к медикаментозным средствам заболеваний (экзема, псориаз, нейродермиты и др.), в аллергологических центрах для комбинированной терапии полиэтиологичных аллергических реакций $[3,4]$.

Общеизвестно, что при кратковременном контакте тела человека с воздухом, охлажденным менее чем до $-110^{\circ} \mathrm{C}$, возникает кратковременно выраженный температурный стресс [2,5]. В процессе воздействия экстремального холода температура кожи человека кратковременно снижается до $+(10-20)^{\circ} \mathrm{C}$. В результате этого включаются в работу абсолютно все терморецепторы кожи, перестраиваются сложнейшие механизмы теплопродукции и теплоотдачи в организме, усиливается взаимодействие различных отделов центральной нервной системы, сердечно-сосудистой и дыхательной систем, стимулируется работа желез внутренней секреции, что, в конечном счете, ведет к перестройке характера обмена веществ в организме $[2,6]$. При этом замедляется скорость проведения болевых нервных импульсов, а при температуре кожи $+5^{\circ} \mathrm{C}$ может наступить полная их блокада [2]. Основным преимуществом таких взаимодействий является, в первую очередь, выраженный (длительностью около 3 часов) обезболивающий эффект $[3,7]$.

Клиническую эффективность воздушной криотерапии чаще всего ставят в зависимость от влияния различных факторов $[2,5,7]$. С одной стороны, эффективность криотерапии зависит от характеристик температурного воздействия (вид воздействия и его интенсивность, длительность и периодичность криовоздействия, площадь охлаждаемой поверхности кожи, интервал между воздействиями), а с другой - от индивидуальных особенностей пациента (вид патологии и фаза заболевания, возраст, пол, переносимость низкой температуры, наличие сопутствующих заболеваний и др.) $[1,4]$. Для достижения максимальной клинической эффективности данного физиотерапевтического метода при проведении курсового лечения многими авторами предлагаются разные подходы $[4,5,7]$. Чаще всего в повседневной клинической практике вид, количество и продолжительность сеансов лечебного низкотемпературного воздействия рассчитываются либо на основе субъективных ощущений пациента, либо на основе результатов объективных данных состояния сердечнососудистой системы больного [2,3]. На сегодняшний день проблема оптимального выбора периодичности и длительности воздействия экстремально низкой температуры на пациентов с болевыми синдромами различной этиологии остается дискутабельной, требует дальнейшего изучения и методологического совершенствования $[1,5]$.
Цель исследования - провести оценку обезболивающего эффекта и влияющих на него факторов при воздействии экстремально низкой температуры в условиях криокамеры для общей воздушной криотерапии.

\section{Материалы и методы}

В исследовании добровольно принимали участие 335 пациентов (144 мужчины и 191 женщина) в возрасте от 20 до 72 лет с патологией опорно-двигательного аппарата (деформирующие дорсопатии) с доминирующим в клинической картине болевым синдромом. Исследуемые на момент начала криолечения не принимали каких-либо медикаментозных обезболивающих средств. Все пациенты были разделены на 4 возрастные группы: в первую группу вошли больные в возрасте от 20 до 35 лет (15 мужчин, 40 женщин), вторую - составили пациенты возрастной категории от 36 до 45 лет (18 мужчин и 54 женщины), третью - в возрасте от 46 до 60 лет (93 мужчины и 46 женщин), и четвёртую - от 61 до 72 лет (18 мужчин и 51 женщина). Для исследования применялась воздушная групповая криокамеpa «Zimmer Medizin Systeme» (Германия), которая способна генерировать экстремально низкие температуры воздуха в пределах от $-10^{\circ} \mathrm{C}$ до $-110^{\circ} \mathrm{C}$.

До проведения курса общей криотерапии были выполнены клинико-лабораторные и инструментальные обследования с целью выявления лиц с абсолютными противопоказаниями для холодового воздействия, к которым относятся гипертоническая болезнь, перенесенный инфаркт миокарда либо мозга (в течение последних 6 месяцев), острые декомпенсированные состояния со стороны внутренних органов и систем, тяжелые хронические заболевания сердечно-сосудистой системы, органов дыхания, нервной системы, мочевыделительной системы в стадии обострения, выраженная патология артериальных сосудов (васкулиты, артерииты, болезнь Рейно), тромбоэмболическая патология магистральных сосудов [2,5,7]. Обязательным условием участия в исследовании было подписание информированного согласия пациента, при этом были соблюдены этические принципы проведения клинических испытаний и положения Хельсинской декларации Всемирной Медицинской Ассоциации, принятой Генеральной ассамблеей Всемирной медицинской ассоциации (1997-2000 гг.).

Непосредственно до и после сеансов криотерапии каждого пациента осматривали физиотерапевт и невролог, проводились термометрия, пульсовая диагностика и измерение артериального давления. Основным методом определения эффективности лечения являлась экспертная оценка выраженности клинической симптоматики [6]. Дополнительным методом при этом было применение визуально-аналоговых шкал «VAS» выраженности ведущих симптомов [8]. В дальнейшем полученные результаты оценивались по разработанной интегральной шестибалльной шкале выраженности обезболивающего эффекта (шкала Лайкерта без средней нулевой точки). В данной интегральной оценочной шкале значение условного показателя «-3» соответствовало отсутствию какого-либо минимального улучшения в состоянии исследуемого, а «+3» максимально выраженному противоболевому эффекту [1]. 
При подборе схемы общего аэрокриовоздействия учитывались рекомендуемые в практике физиотерапевтического лечения методики для данной патологии $[2,5]$. Стандартная продолжительность курса составляла не менее 40 ежедневных сеансов. При необходимости курс лечения или сеанс могли быть прерваны по просьбе пациента или объективным причинам.

Математический анализ полученных данных проводился в статистических пакетах прикладных программ «MedCalc v. 11.6.0.0» (MedCalc software bvba) и «Statistica 6.0» (StatSoft, Inc). Для статистической обработки результатов исследований были применены базовые методы математической статистики: описательная статистика, парные и множественные сравнения, регрессионный анализ. При этом первым этапом обработки данных была проверка принадлежности данных к нормальному закону распределения случайных величин путем проверки нулевой гипотезы с использованием критерия Шапиро-Уилки (при $\mathrm{n}<30$ ) или Хи-квадрат (при n>30). При парном сравнении использовались параметрические (критерии Стьюдента и Фишера) или непараметрические (W-критерии Уилкоксона и Хи-квадрат) критерии. При сравнении трех или более групп были использованы метод однофакторного дисперсионного анализа Крускала-Уоллиса и методы множественных сравнений Шеффе или Дана. Учитывая тот факт, что анализируемые данные являются цензурированными, для оценки доли пациентов, у которых событие (максимальный обезболивающий эффект «+3» по интегральной шкале) не произошло, применялся описательный метод Каплана-Майера [9]. Для оценки достоверности различий среди кривых вероятности отсутствия обезболивающего эффекта были использованы методы сравнения кривых выживаемости: логранк тест (log-rank test) или модель пропорциональных интенсивностей Кокса (Cox proportional-hazards regression) [9]. Во всех случаях в качестве критического уровня значимости принималось значение 0,05.

\section{Результаты и обсуждение}

Исходное состояние организма пациентов характеризовалось 100\%-ным доминированием болевого синдрома при анализируемой клинической патологии. Учитывая, что достижение максимального обезболивающего эффекта у пациентов по шкале интегральной оценки соответствует баллу «+3», этот рубеж был предложен как основное событие при использовании метода построения и оценки кривых «выживаемости». В результате проведенных расчетов были рассчитаны и построены кривые вероятности отсутствия обезболивающего эффекта в динамике курса для разных возрастных групп, что изображено на рис.1.

До начала проведения сеансов воздушной аэрокриотерапии у $27,9 \pm 6,8 \%$ исследуемых болевые ощущения были максимально выраженными и оценивались по интегральной шкале на уровне «-3» балла, а у 72,1 \pm 6,8\% пациентов болевые ощущения были умеренно сильные и оценивались на уровне «-2» балла. Пациентов без болевого синдрома в этот период не было. Статистически значимых возрастных межгрупповых различий при оценке степени достижения противоболевого эффекта не выявлено (Chi-square $=2,53 ; \mathrm{p}=0,47$ ).
В динамике курса лечения к 10-му дню во всех исследуемых группах было отмечено увеличение количества пациентов с интегральной оценкой «+3». Так, в I, II и III группах удельный вес больных с максимально выраженным обезболивающим эффектом составил $36,4 \pm 6,5 \%, 36,1 \pm 5,7 \%$ и $34,5 \pm 4,0 \%$, соответственно. В IV группе доля таких пациентов составила $24,6 \pm 5,2 \%$. Однако при проведении множественных сравнений статистически значимых межгрупповых различий выявлено не было (Chi-square $=2,04 ; \mathrm{p}=0,56$ ).

На 15-й день курсового лечения обобщенная межгрупповая доля больных, у которых проявился устойчивый обезболивающий эффект, составила 58,1 \pm 7,5 \%. В этот период лечения межгрупповых различий также выявлено не было (Chi-square=1,38; p=0,58).

Анализ расположения кривых вероятности отсутствия обезболивающего эффекта у больных с дорсопатиями (рис.1) показал, что медиана «выживаемости» (50,0\% обследуемых) приходилась ориентировочно на $21,0 \pm 2,0$ сеанс курсового лечения, при этом обобщенный удельный вес пациентов с баллом «+3» по интегральной шкале в этот период лечения составил 74,3 $\pm 7,4 \%$, а с баллом «+2» и «+1»-8,6 4 4,7\% и 17,1 $6,4 \%$, соответственно (Chi-square=69,09; p<0,001). Heобходимо отметить, что выраженность обезболивающего воздействия при общей экстремальной воздушной криотерапии в каждой группе изменялась постепенно и достигала максимального удельного веса $(97,1 \pm 4,1 \%)$ после $30,0 \pm 1,0$ сеанса, при этом не зависела от возрастных характеристик исследуемых групп (Chi-square=0,27; p=0,42). Выявленные особенности обезболивающего воздействия подтверждают научные результаты Владимирова А.А. и соавт., указывающих, что для достижения оптимального анальгетического эффекта у больных с остеохондрозом позвоночника целесообразно применение общей воздушной криотерапии в течение 3 недель [10].

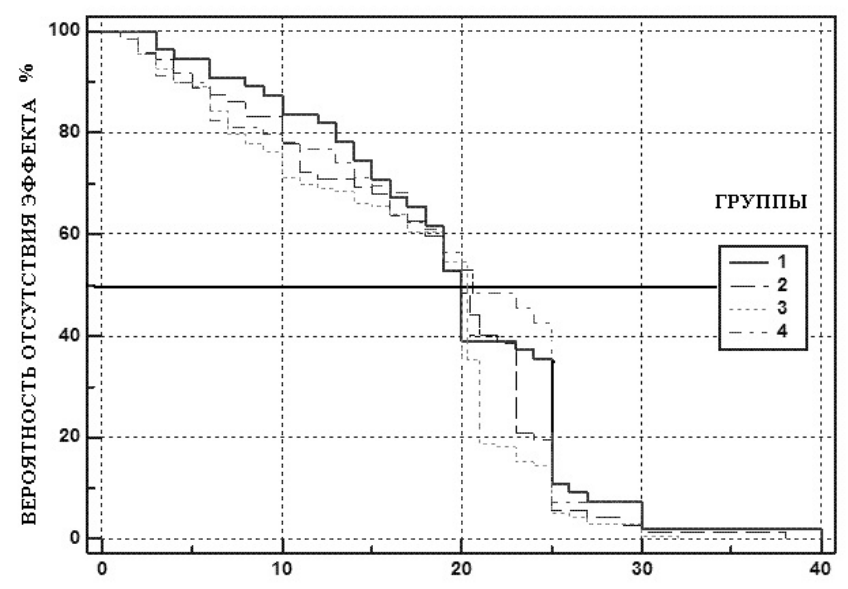

Рис. 1. Кривые вероятности отсутствия обезболивающего эффекта в разных возрастных группах при воздействии экстремально низкой температуры.

Примечание: 1-2-3-4 - возрастные группы; под основным событием понималось достижение выраженного обезболивающего эффекта на уровне «+3» балла по интегральной шкале. 
По данным некоторых авторов [1,2,3], существуют доказанные различия терморегуляторных реакций у физически здоровых мужчин и женщин. По данным Бердышева Г.Д. и соавт., у мужчин интенсивнее осуществляется деятельность сердца и выделение пота при нейтральной или повышенной внешней температуре, чем у женщин, при отсутствии различий кожной и ректальной температуры. Кратковременное охлаждении тела у женщин приводит к меньшему замедлению метаболических процессов, чем у мужчин $[1,3]$. Однако при воздействии экстремально низкой температуры эти различия могут не проявляться, особенно у пациентов с имеющейся сопутствующей возрастной патологией сердечно-сосудистой системы, при которой даже непродолжительный сосудистый спазм может привести к появлению клинических признаков обострения заболевания, а иногда даже к ишемическому повреждению тканей [3]. При этом, очевидно, что время однократной криотермической процедуры может быть для таких пациентов уменьшено, а длительность курса лечения пролонгирована $[2,10]$.

Для проверки гипотезы гендерного влияния на эффективность лечения у больных с деформирующими дорсопатиями была рассчитана функция вероятности отсутствия лечебного эффекта и построены кривые «выживаемости» с учетом гендерных различий (рис. 2).

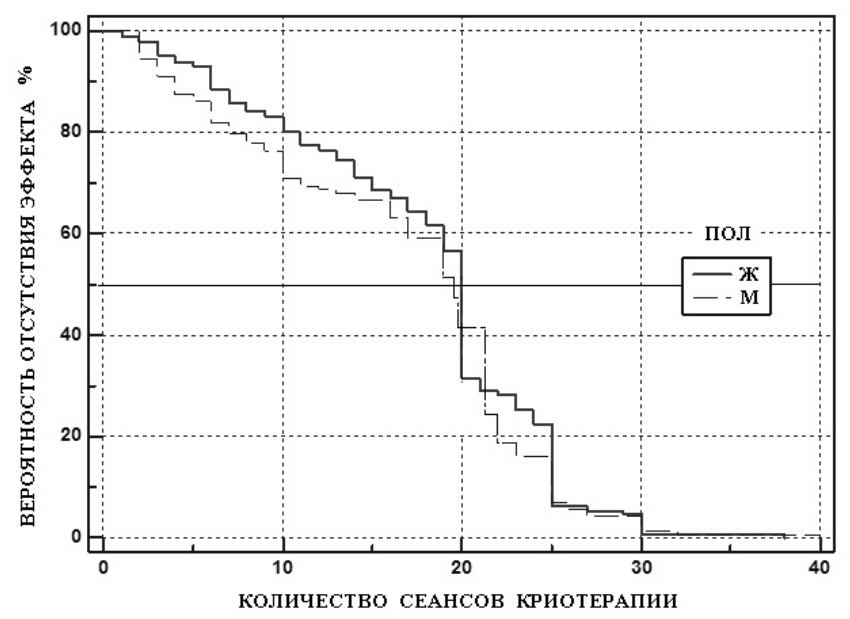

Рис. 2. Кривые вероятности отсутствия обезболивающего эффекта в разных гендерных группах при воздействии экстремально низкой температуры.

Примечание: м - мужчины, ж - женщины; под основным событием понималось достижение выраженного обезболивающего эффекта на уровне «+3» балла по интегральной шкале.

В результате проведенного анализа было выявлено, что на 10-й день курса удельный вес больных с выраженным обезболивающим эффектом («+3» балла по интегральной шкале) среди женщин увеличился от 0 до

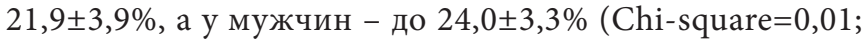
$\mathrm{p}=0,94)$.

Анализ кривых вероятности отсутствия обезболивающего эффекта среди мужчин и женщин показал, что медиана убывания доли пациентов, не достигших

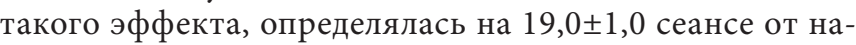
чала курсового воздействия. Статистически значимых межгрупповых различий при оценке срока достижения противоболевого эффекта у мужчин и женщин выявлено не было (Chi-square $=1,74 ;$ при $\mathrm{p}=0,187$ ). При расчете показателей лог-ранк теста была подтверждена нулевая гипотеза об отсутствии статистически значимых различий между кривыми вероятности отсутствия обезболивающего эффекта у мужчин и женщин (критерий $\mathrm{z}=2,06 ; \mathrm{p}<0,05)$. Максимальный удельный вес пациентов $(96,3 \pm 2,3 \%)$ с выраженным обезболивающим эффектом определялся не ранее $30,0 \pm 0,5$ сеанса, независимо от гендерных признаков пациентов.

Таким образом, для достижения максимального обезболивающего эффекта у половины наблюдаемых больных потребовалось не менее 20 сеансов аэрокриовоздействия независимо от возраста пациентов и гендерных различий.

Для определения степени влияния ряда независимых факторов (сезонности, гендерных различий, возраста) на выраженность обезболивающего эффекта использовался метод построения многофакторной регрессии (Cox proportional-hazards regression), который позволил оценить степень эффективности криовоздействия через отношение рисков недостижения обезболивающего эффекта. Данные многофакторной модели, учитывающей сезонность, пол исследуемых и их возраст, приведены в табл.1.

Как видно из табл.1, влияние фактора сезонности на эффективность обезболивающего лечения экстремально низкой температурой определялось как незначительное, т.к. коэффициенты модели, характеризующие соотношение летнего и других периодов года, принимали значения от $0,06 \pm 0,16$ (лето-осень) до $0,25 \pm 0,16$ (лето-зима) при уровне статистической значимости, превышающей критическое значение $(p>0,05)$. Отношение рисков по факторам взаимоотношения сезонов года находилось в пределах от 1,0636 до 1,2869 (р>0,05), что говорит о слабой взаимосвязи данного фактора с исходом и соответствует не более $51,5 \%$ - 56,3\% шанса раннего наступления обезболивающего эффекта у пациентов в теплый период года, чем в холодный или умеренно-холодный. Это свидетельствует об отсутствии влияния фактора сезонности на степень обезболивающего эффекта у данного контингента больных, что совпадает с результатами исследований ряда авторов $[2,4,5]$.

Аналогичные результаты отмечались при изучении влияния гендерных и возрастных факторов на степень обезболивающего эффекта при воздействии экстремально низкой температуры. Так, коэффициенты модели, характеризующие гендерные и возрастные факторы, приближаются к нулю (р>0,1 во всех случаях), границы $95 \%$ ДИ отношения рисков включают значение 1,0 , что достоверно указывает на отсутствие статистически значимых влияний изучаемых факторов на степень выраженности обезболивающего эффекта. Отсутствие влияния возрастных факторов у больных с дорсопатиями на уровень анальгетического эффекта совпадают с результатами Pournot Н. и Braun K.P. [5, 7].

Таким образом, проведенный анализ и оценка обезболивающего эффекта экстремально низкой температуры показали, что минимальное количество сеансов, необходимых для формирования стойкого эффекта минимум у

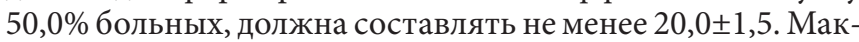
симальный удельный вес пациентов (более 95\% больных) с выраженным обезболивающим эффектом формируется 
Таблица 1.

\section{Статистические характеристики многофакторной модели, определяющей зависимость обезболивающего эффекта от изучаемых факторов у больных с деформирующими дорсопатиями при воздействии экстремально низкой температуры}

\begin{tabular}{|l|c|c|c|c|c|}
\hline \multicolumn{1}{|c|}{ Факторы } & $\mathrm{b}$ & $\pm \mathrm{m}$ & ОР & 95\% ДИ ОР & $\mathrm{p}$ \\
\hline Лето - осень & 0,0617 & 0,1599 & 1,0636 & 0,7787 to 1,4528 & 0,6996 \\
\hline Лето - весна & 0,1958 & 0,1446 & 1,2163 & 0,9174 to 1,6125 & 0,1757 \\
\hline Лето - зима & 0,2523 & 0,1636 & 1,2869 & 0,9355 to 1,7705 & 0,123 \\
\hline Гендерный фактор & 0,1677 & 0,1423 & 1,1826 & 0,8960 to 1,5609 & 0,2385 \\
\hline Возраст & $-0,01356$ & 0,05669 & 0,9865 & 0,8833 to 1,1018 & 0,811 \\
\hline
\end{tabular}

Примечание: $\mathrm{b}$ - коэффициенты модели для каждого из факторов; $\pm \mathrm{m}$ - стандартная ошибка коэффициентов модели; ОР - отношение рисков; 95\% ДИ ОР - 95\% доверительный интервал отношения рисков; р - уровень статистической значимости.

лишь после 30-го сеанса криотерапевтического курсового лечения. При этом степень эффективности обезболивающего воздействия у пациентов с деформирующими дорсопатиями не зависит от влияния возраста, гендерных различий или фактора сезонности ( $>>0,05)$.

\section{Выводы}

При воздействии экстремально низкой температуры на организм пациентов с заболеваниями опорно-двигательного аппарата (деформирующие дорсопатии) обезбо- ливающий эффект в динамике 40-дневного курса криолечения проявляется постепенно, достигая максимального значения более чем у половины обследуемых пациентов

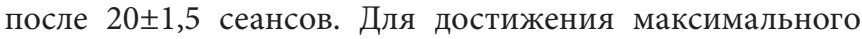
лечебного обезболивающего эффекта у 95\% больных потребуется не менее 30-ти ежедневных сеансов общей воздушной криотерапии в условиях криокамеры.

Влияния возрастных, гендерных и сезонных факторов на эффективность противоболевого лечебного воздействия у больных с деформирующими дорсопатиями статистически не выявлено ( $\mathrm{p}>0,05)$.

\section{ЛИТЕРАТУРА}

1. Лях Ю.Е. Количественная оценка эффекта воздействия общей экстремальной воздушной криотерапии на организм человека / Ю.Е. Лях, В.Г.Гурьянов, С.М.Тетюра, С.М.Радченко // Університетська клініка. - 2012. - Т.8, № 1. - С.13-16.

2. Papenfuв W. Power from the Cold / Winfried Papenfus. - Edition k, holder: Friedrich Kehrer, Regensburg. - 1st ed. (in English), 2006. $-142 \mathrm{p}$.

3. Бердышев Г.Д. Медико-технические основы термологии / Г.Д. Бердышев, Н.М. Уланов. - Киев: Фитосоциоцентр, 2006. $379 \mathrm{c}$.

4. Effectiveness of different cryotherapies on pain and disease activity in active rheumatoid arthritis. A randomised single blinded controlled trial / H.E. Hirvonen, M.K. Mikkelsson, H. Kautiainen [et al.]. - Leirisalo-Repo, 2006. - P. 67.

5. Pournot H. Time-course of changes in inflammatory response after whole-body cryotherapy multi exposures following severe exercise / H. Pournot // PLoS One. - 01-JAN-2011.- № 6(7). P. 227-248.
6. Breivik H. Assessment of pain. / H. Breivik, P.C. Borchgrevink, S.M. Allen et al. // British Journal of Anaesthesia. - 2008. - № 101 (1). - P. 17-24.

7. Whole-body cryotherapy in patients with inflammatory rheumatic disease / K.P. Braun, S. Brookman-Amissah, K. Geissler [et al.] // Med. Klin. (Munich). - 2009. - Vol. 15, № 3. P. 192-198.

8. Wewers M.E., Lowe N.K. A critical review of visual analogue scales in the measurement of clinical phenomena / Res Nurs Health. - 1990. - № 13. - P. 227-236.

9. Petrie A. Medical statistics at a glance / Aviva Petrie, Caroline Sebin. - Replika Press Pvt. Ltd, India. - 2nd ed., 2005. - 160 p.

10. Владимиров А. А. Использование общей криотерапии для снятия болевого синдрома у пациентов с остеохондрозом позвоночника в период обострения / А. А. Владимиров, Г. А. Гурбич, Д. Г. Измайлов // Криотерапия: безопасные технологии применения: сборник статей / под общ. ред. д-ра мед. наук, проф. О.А. Панченко. - К.: КВИЦ, 2012. - С. 28-32. 